\title{
Появление первых теорий адсорбции в 20 веке
}

\author{
Панарин В.Ю., Баум Е.А., Ланин С.Н. \\ Московский государственный университет им. М.В. Ломоносова, Москва
}

Поступила в редакцию 31.10.2018 г.

DOI: https://doi.org/10.17308/sorpchrom.2019.19/754

В статье дан исторический аспект появления первых теорий адсорбции в 20 веке: с одной стороны, потенциальных теорий А.Т. Эйкена и М. Полани (1914), а с другой стороны мономолекулярной теории И. Ленгмюра (1916-1918). Продемонстрированы основные положения этих теорий в аналитическом контексте.

Ключевые слова: история химии, адсорбция

\section{The appearance of the first adsorption theories in the 20th century}

\author{
Panarin V.Y., Baum E.A., Lanin S.N. \\ Lomonosov Moscow state university, Moscow
}

In the 19th century, the adsorption of gases by coal was studied in detail, but there were difficulties in comparing the experimental data, because the studies have not been standardized. At the beginning of the 20th century, much attention was paid to the reproducibility of the experiment, which made it possible to compare the experimental data among themselves, to find certain dependencies, and to create theories of adsorption. In 1914-1918, independently of each other, two theories of adsorption were proposed - the potential theory of A. Eucken and M. Polanyi and the monomolecular theory of I. Langmuir. The historical context of the indicated theories and their main provisions are shown.

A. Eucken, using the works of W.H. Nernst in the field of thermodynamics and electrochemistry, proposed a potential theory of adsorption. He suggested the existence of the «atmosphere» of adsorbed gas in a compressed state that adsorbed molecules, which do not interact with each other and that the interaction between the adsorbent and molecules is expressed by the adsorption force of attraction and depends on the distance between them.

M. Polanyi, also based on the works of W.H. Nernst, suggested that the van-der-Waals forces act between the adsorbent and the adsorbate, which form layers on the surface of a solid, and the gas obeys the laws of thermodynamics. If the force of attraction between the adsorbent and the gas compresses the gas to a certain extent, then it condenses on the surface of the adsorbent. In this case, the adsorbed gas can have a thickness of several molecules on the surface of the adsorbent, and the adsorbate molecules are located on the surface in a certain order. In this case, neither Eucken nor Polanyi proposed an equation for the adsorption isotherm. This was done by Langmuir when he created a new theory of adsorption.

I. Langmuir in 1916-1918 introduced the concept of monolayer adsorption on energetically homogeneous solid surfaces and derived an equation for the adsorption isotherm obtained from kinetic studies. Langmuir suggested the occurrence of electrostatic forces during the adsorption process and used in his works the G.N. Lewis's electron-pair theory of chemical valence (1916). In this respect, his approach was innovative for that time.

Langmuir's theory received support of many scientists, as it was in good agreement with thermodynamics and with the theory of intermolecular forces of Debye-Hückel. Supporters of his theory was F. Haber, A. Einstein. The latter were at the same time opposed to the theory of Polanyi, believing that his theory was not sufficiently well founded. Supported the theory of Polanyi H.M. Freundlich and several other scientists. 
However, Freundlich believed that by that time an enough experimental facts had not been accumulated that would unequivocally testify in favor of one of them (all the theories are correct).

At the beginning of the 20th century, after a long period of accumulation of experimental material on adsorption in the 19th century, a period of active creation of adsorption theories came. Their appearance was facilitated by advances in the development of chemistry and physics in the early 20th century, related to the development of chemical thermodynamics and theories of chemical bonding.

Keywords: history of chemistry, adsorption

\section{Введение}

В 19 веке было очень подробно изучено явление адсорбции газов углём и накоплено много экспериментального материала по адсорбции газов различными видами угля. Однако исследователи не смогли его использовать для создания теорий адсорбции. Было установлено, что адсорбция зависит от вида угля (растительный или животный), способа его получения, вида древесины, из которой он был получен, а также от степени измельченности угля (Н.Т. де Соссюр, П. Шаппюи, Г. Магнус, Г. Кайзер и др.) [1-9]. При этом очень сложно было сопоставлять экспериментальные данные, так как исследования не были стандартизованы. Именно в начале 20 века при изучении адсорбции стали уделять большое внимание воспроизводимости эксперимента. Проведение экспериментов в одинаковых условиях и, следовательно, хорошая их воспроизводимость предоставили возможность сравнивать между собой опытные данные, находить определенные зависимости и создавать теории адсорбций.

В начале 20 века природа адсорбции газов была основным предметом изучения большинства экспериментаторов. Научная задача состояла в том, чтобы описать явление адсорбции газов и объяснить изотермы в терминах новой науки - физической химии, сформированной к концу 19 века, как самостоятельной области знаний. В 1914-1918 годах появились два независимых описания адсорбционных явлений, связанных с такими именами, как А.Т. Эйкен и М. Полани, с одной стороны, и И. Ленгмюр, с другой [10-28].

\section{Потенциальная теория адсорбции А.Т. Эйкена (1914)}

Немецкий химик и физик Арнольд Томас Эйкен (1884-1950) родился в Йене (Германия) и был сыном философа, а затем и лауреата Нобелевской премии по литературе (1908) Рудольфа Кристофа Эйкена (1846-1926). Арнольд Эйкен учился в Киле, Йене, а затем в Берлине, где с 1905 года работал в Берлинском Университете под руководством физикохимика Вальтера Германа Нернста (1864-1941), разработавшего как раз к тому времени свою знаменитую «тепловую теорему», известную теперь как «третье начало термодинамики» (1905). Отталкиваясь от работ Нернста в области термодинамики и электрохимии, Эйкен создал потенциальную теорию адсорбции.

Согласно Эйкену [10] над поверхностью адсорбента находится «атмосфера» адсорбированного газа в сжатом состоянии. Предполагается, что адсорбированные молекулы не взаимодействуют между собой и что взаимодействие между адсорбентом и молекулами выражается адсорбционной силой притяжения и зависит от расстояния между ними.

Ученый предположил, что адсорбционные силы не зависят от температуры [11], адсорбция какой-либо одной молекулы газа не зависит от других уже адсорбированных молекул и молекулы в адсорбированном слое подчиняются одному и тому же уравнению состояния для реальных газов (уравнению Ван-дер-Ваальса, 1873 г.), так, словно они не адсорбируются [11]. Предложенная модель позволила вывести 
уравнения, описывающие адсорбцию идеального и реального газа плоской поверхностью. Коллеги А. Эйкена, в частности, британские исследователи Е. Свэн и А.Р. Уркварт (1926) утверждали, что его теория хорошо согласуется с экспериментальными данными [12].

\section{Потенциальная и полимолекулярная теория адсорбции М. Полани (1914)}

Майкл Полани (1871-1976) заинтересовался физической и коллоидной химией при изучении адсорбции коллоидными гелями в лаборатории венгерского физиолога и патологоанатома Ференца Тангла (Ferenc Tangl, 1866-1917), когда был студентом-медиком в Будапеште. Там, во время проведения медицинских исследований в 1908-1913 годах, Полани занимался экспериментальными работами в области биохимии (Институт патологии и физиологической химии). В лаборатории он изучал множество различных вещей, в том числе применимость второго закона термодинамики к организмам животных или некоторые свойства коллоидных веществ, такие как набухание, осмотическое давление и адсорбция. Уверенность Тангла в том, что физиология должна основываться на глубоких знаниях физической химии, способствовала интересу Полани к этой области. Полани погрузился в термодинамику, физическую химию и физику, читал учебник В.Г. Нернста по теоретической физической химии, из которого узнал о тепловой теореме Нернста (1905). Из книги Нернста Полани почерпнул теоретические основы для своих научных исследований. В 1913 году наконец-то он получил медицинский диплом Будапештского университета. В каникулярное время Полани посещал летние курсы по физической химии, электрохимии, контактной (поверхностной) химии и катализу в Высшей Политехнической Школе в Карлсруэ, где познакомился со своим будущим преподавателем Георгом Бредигом (1868-1944), выдающимся исследователем в области термодинамики. В 1913 году Полани поступил в Высшую Политехническую Школу в Карлсруэ в качестве студента химии под руководством своего преподавателя Бредига (19131914 гг.). Во время Первой мировой войны в 1914-1915 годах Полани был вынужден вернуться в Австро-Венгрию. Там он служил в австро-венгерской армии медицинским офицером, однако его интерес к физической химии продолжал ярко проявляться в течение этого периода и он написал несколько научных работ. Три из его работ были связаны с выводом третьего закона термодинамики (теорема Нернста), а четвертая касалась адсорбции газов твердыми телами. Последняя работа стала его докторской диссертацией по физической химии, которую он защитил в 1917 году на химическом факультете Будапештского университета, получив докторскую степень по химии [13-15]. Эта диссертация, написанная ученым во время войны (19141916 гг.), была сфокусирована на адсорбции газов на поверхности коллоидных капель и пористых твердых тел [16].

Полани предположил, что между адсорбирующим телом и атомами или молекулами газа, образующими слои на поверхности твердого тела, существуют силы межмолекулярного притяжения типа Ван-дер-Ваальса, которые являются слабыми силами притяжения между атомами или неполярными молекулами. Согласно Полани, газ подчиняется законам термодинамики, и силы Ван-дер-Ваальса действуют между неполярными атомами [11]. Если сила притяжения сжимает газ до определенной степени, то он конденсируется на поверхности адсорбента. При этом адсорбированный газ может иметь толщину в несколько молекул на поверхности адсорбента, и эти молекулы адсорбата расположены в определенном порядке [11].

Еще, будучи студентом и работая в лаборатории Тангла в Будапеште, как уже нами отмечалось выше, Полани узнал о тепловой теореме Нернста из его учебника 
(третьем законе термодинамики (1905)), и у него появилась определенная идея, как использовать этот закон для создания собственной теории адсорбции. В 1914 году Полани, теоретически [17], с применением третьего закона термодинамики $\left(\Delta \mathrm{S}_{\mathrm{T} \rightarrow 0} \rightarrow 0\right)$, предположил, что энтропия адсорбционной системы будет уменьшаться до нуля при бесконечно высоких давлениях, по аналогии со стремлением энтропии к нулю при понижении температуры до нуля из рассуждений В. Нернста. При стремлении давления к бесконечности все больше ограничивается движение молекул и тем самым уменьшается энтропия до нуля при бесконечном давлении. Поэтому, при низких температурах или высоких давлениях адсорбционная система будет себя вести как идеальный концентрированный раствор, а с ростом температуры или при понижении давления ее поведение будет постепенно приближаться к поведению разбавленного реального раствора. Полани предположил, что при поглощении газов адсорбентом адсорбированный слой поглощенного вещества сильно сжимается молекулярными силами и что молекулярная сила изменяется с расстоянием по экспоненциальной зависимости [12].

Полани считал, что потенциальная энергия притяжения зависит от расстояния между поверхностью и газовой частицей, но она не зависит от наличия других частиц газа в поле адсорбционного потенциала. Поэтому, он записал выражение для адсорбционного потенциала (энергии сродства адсорбата к адсорбенту), как меры интенсивности адсорбционного взаимодействия, в виде зависимости адсорбционного потенциала от адсорбционного объема, в котором эта сила притяжения эффективна. Он показал, что адсорбционный потенциал при постоянном объеме жидкого адсорбата на адсорбенте (постоянной степени объемного заполнения) не зависит от температуры [11].

Чуть позже, в работе 1922 года Полани утверждает превосходство своей теории над теорией Эйкена. Полани обнаружил, что выражение для теплоты адсорбции у Эйкена подходит для описания газа в условиях, когда он не конденсируется, однако для газов в условиях, когда они конденсируются, в это уравнение также необходимо добавить молярную теплоту испарения, что и сделал Полани [18].

Адсорбционные силы в теориях Эйкена и Полани - физические, Ван-дерВаальсовы взаимодействия и не зависят от температуры, а также в их теориях не учитывается взаимодействие молекул адсорбата между собой. Теория Полани, на самом деле, является расширением теории Эйкена. Полани более подробно описывал действие адсорбционных сил. Он считал, что со стороны газовой фазы действует силовое поле, которое возникает с помощью межмолекулярных сил, в котором конденсируются молекулы адсорбата. Эйкен использовал термин «адсорбционные силы”, а Полани дал определение адсорбционным силам через адсорбционный потенциал и потенциальный энергетический градиент. Полани определил адсорбционный потенциал в точке вблизи адсорбента как работу адсорбционных сил по доставке молекулы из газовой фазы к этой точке. Эта работа адсорбционных сил представляла собой работу сжатия при конденсации пара. При этом первый слой адсорбата находился под наибольшим сжатием, второй слой при меньшем сжатии и т. д., до тех пор, пока плотность газа не уменьшится до плотности окружающего газа. Таким образом, существует градиент потенциальных поверхностей от $\varepsilon_{0}, \varepsilon_{1}, \varepsilon_{2} \ldots$ до $\varepsilon_{\text {i }}$, а плотность адсорбированного слоя уменьшается с удалением от поверхности твердого тела [11].

Полани считал, что адсорбционные силы действуют на больших расстояниях, чем размер молекул адсорбата, то есть у поверхности существует адсорбционный объем, заполненный молекулами адсорбата. И одно из самых важных положений его теории состоит в том, что притяжение молекул поверхностью адсорбента не зависит 
от наличия в адсорбционном пространстве других молекул, что делает возможной полимолекулярную адсорбцию. Однако, ни Эйкен, ни Полани не предложили никакого обобщенного уравнения для изотермы адсорбции, из которой по одной экспериментальной изотерме можно было бы теоретически вычислить другие изотермы при разных температурах [11]. Однако это удалось сделать Ленгмюру при создании им новой теории адсорбции.

Идея простого притяжения между адсорбентом и газом была чужда большинству ученых того времени. В то время были известны только два типа сил, действующих между атомами: электрические и валентные силы. Валентные силы насыщаются одним адсорбированным слоем, а электрические силы зависят от заряда. Ни одна из этих двух сил не могла бы способствовать образованию нескольких слоев газа, и считалось, что только один слой молекул или атомов может быть адсорбирован на поверхности адсорбента. В это предположение верили многие ученые того времени. Явление адсорбции является очень сложным, поскольку существует несколько типов адсорбционных сил. Возможно, еще до 1930 года Полани предполагал, что существуют различные виды сил, которые могут быть ответственны за адсорбцию, и что некоторые из этих сил не соответствуют его теории. Идея простого адсорбционного потенциала, предложенная Полани, нашла теоретическое обоснование в теории дисперсионных сил Уонга-Лондона (Wong W.A., London F.W.) (1927 и 1930), объясняющих притяжение и отталкивание между атомами. Совместная публикация немецкого физика-теоретика Фрица Вольфганга Лондона (Fritz Wolfgang London, 1900-1954) и Полани в 1930 году [19] показала, что дисперсионные силы удовлетворяют предположениям теории Полани в очень хорошем приближении. Учёные предложили новое обобщенное объяснение адсорбционных сил (дисперсионных сил) с использованием квантовой механики.

\section{Мономолекулярная теория адсорбции И. Ленгмюра (1916-1918)}

Американский физикохимик Ирвинг Ленгмюр (1881-1957) обучался в Гёттингенском университете (1903-1906) и был учеником В.Г. Нернста, будущего лауреата Нобелевской премии по химии (1920), что сыграло ключевую роль в его выдающихся научных достижениях. В 1906 году Гёттингенским университетом ему была присуждена докторская степень по физике. С 1909 работал в лаборатории «Дженерал Электрик» (General Electric Laboratory) в Скенектади (штат Нью-Йорк), и в 1916-1918 годах опубликовал экспериментальные результаты по адсорбции газов на слюдяных поверхностях, стекле и платине.

В своих работах, выполненных в эти годы, он утверждал, что поверхностный слой мономолекулярен в соответствии со структурой, определяемой электростатическими силами, и ввел четкое понятие монослойной адсорбции, сформированное для энергетически однородных твердых поверхностей. Ленгмюр отклонил предположение о том, что при адсорбции может иметь место образование нескольких слоёв адсорбированных газов. Уравнение Ленгмюра, первоначально полученное из кинетических исследований, основывалось на предположении, что на поверхности адсорбента имеется определенное и энергетически эквивалентное число адсорбционных центров, при этом на каждом из них может адсорбироваться одна молекула идеального газа. Связывание с центрами адсорбции может быть как химическим, так и физическим, но оно должно быть достаточно сильным, чтобы предотвратить смещение адсорбированных молекул вдоль поверхности. Таким образом, Ленгмюр впервые выдвинул четкое представление о мономолекулярной адсорбции на энергетически однородных поверхностях [20-22].

Панарин и др. / Сорбционные и хроматографические процессы. 2019. Т. 19. № 3 
Ленгмюр предположил возникновение электростатических сил при адсорбции и использовал в своих работах новую электронно-парную теорию химической валентности, которая была сформулирована американским физико-химиком Гилбертом Ньютоном Льюисом (1875-1946) в 1916 году. В этом отношении его подход был новаторским для того времени. Ленгмюр утверждал, что сила, которая удерживает частицы адсорбированного газа на твердой поверхности, возникает из-за электрических валентных сил атомов или молекул в самом внешнем слое твердого тела. Адсорбированные частицы газа укладываются в один слой на поверхности твердого тела, как на шахматной доске, где каждый квадрат или отверстие может быть занято только одной частицей газа. Таким образом, адсорбция прекращается, когда поверхность полностью занята (рис. 1). Теория Ленгмюра представляла собой смелый разрыв с классической термодинамической теорией с предположением о том, что газовый адсорбат (адсорбированный слой) относительно толстый и становится менее плотным по мере увеличения расстояния от поверхности адсорбента [11].

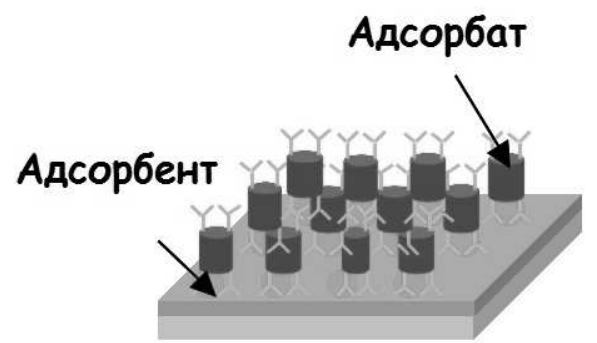

Рис. 1. Схема мономолекулярной адсорбции И. Ленгмюра

Ленгмюр считал, что адсорбция всегда сопровождается противоположным процессом десорбции, удалением частиц газа, которые ранее адсорбировались на поверхности. Между адсорбцией и десорбцией существует динамическое равновесие. Степень заполнения поверхности можно рассчитать по константам скоростей двух обратных процессов. В работе [20] Ленгмюр вывел простое изотермическое уравнение, которое используется и в наше время, для изучения адсорбции. Данное выражение работает при невысоких давлениях и степенях заполнения поверхности. При более высоких давлениях и степенях заполнения используют распространенное уравнение Брунауера-Эммета-Теллера (БЭТ, 1938). Это уравнение изотермы для многослойной адсорбции является развитием теории Ленгмюра на случай полимолекулярной адсорбции.

\section{сорбции \\ Обсуждение и сравнительный анализ рассмотренных теорий ад-}

Сравнивая теории М. Полани и И. Ленгмюра видно, что определяющим фактором является предположение М. Полани о полимолекулярном заполнении поверхности адсорбента адсорбатом и предположение И. Ленгмюра о мономолекулярном заполнении.

Теория Ленгмюра получила огромное признание многих ученых, так как она хорошо согласовывалась, как с термодинамикой, так и с позже возникшей теорией Дебая-Хюккеля (1920-е годы), которая описывает типы и природу возникновения межмолекулярных сил [23]. Его теорию поддерживали такие великие ученые как Ф. Габер, А. Эйнштейн [23] и Г.М. Фрейндлих [24]. В 1932 году Ирвинг Ленгмюр был удостоен Нобелевской премии по химии «за открытия и исследования в области химии поверхностных явлений».

Панарин и др. / Сорбционные и хроматографические процессы. 2019. Т. 19. № 3 
У Полани было множество противников его теории, в том числе Ф. Габер и А. Эйнштейн [13-15]. Первая мировая война закончилась разгромом центральных держав и привела к распаду Австро-Венгрии. Полани оставался в Будапеште, пока не получил докторскую степень по химии (1917). Однако запутанная политическая ситуация, диктаторский характер нового режима, последующие революции и разгар драматического роста антисемитизма побудили его вернуться в Карлсруэ в 1919 году. Именно здесь он заинтересовался проблемой изучения скорости химических реакций. В 1920 году Полани решил переехать в Берлин, стремясь изучать скорость химических реакций, утвердиться в качестве химика, и очень хотел получить перевод в институт Габера. В Берлине его пригласили в Институт химии волокон Общества Кайзера Вильгельма в Берлине для участия в проекте по исследованию волокнистых структур (в частности, целлюлозы) и металлов с использованием новых методов рентгеноструктурного анализа, в том числе метода рентгеновской дифракции. Этот институт находился в том же здании, что и Институт физической химии и электрохимии Общества Кайзера Вильгельма, под руководством Фрица Габера. Его переезд привел к фундаментальным изменениям в его научной деятельности, и привело его к изучению структуры и свойств кристаллов, главным образом, металлов [13$15]$.

В 1921 году Полани представил свою теорию газовой адсорбции на семинаре в институте Габера. Данная тематика была близка Габеру, так как адсорбция тесно связана с гетерогенным катализом, одной из самых важных областей его исследований. Габер пригласил А. Эйнштейна принять участие в семинаре из-за высокой значимости тематики Полани. Оба авторитета, Эйнштейн и Габер, отвергли его теорию. Они не могли принять идею многослойной адсорбции, считали, что его теория не была достаточно хорошо обоснована, не включала в себя знания о современных теориях межмолекулярных взаимодействий (например, теории Дебая-Хюккеля) и электрических теорий строения материй. Полани основывал свою теорию адсорбции на модели классической термодинамики 19 века, потому что не знал о последних электрических представлениях и электронных моделях сил, действующих между атомами и молекулами, появившимися около 1920 года. Однако основные противники Полани - Эйнштейн и Габер, - не работали над альтернативной теорией, в отличие от Ирвинга Ленгмюра, но последний не присутствовал на семинаре. В то же время в семинаре приняли участие также и другие специалисты-эксперты в области науки, сотрудники института Габера, в том числе Герберт Макс Фрейндлих. Они были удовлетворены интерпретацией Полани, тем, как он применяет классический термодинамический подход в своих исследованиях. После неудачного выступления Полани боялся, что потерял свой шанс на перевод в институт Габера. Однако, принимая во внимание работу Полани над кристаллическими структурами, Габер назначил Полани в 1923 году руководителем второго отдела физической химии (химической кинетики) в Институте физической химии и электрохимии имени Кайзера Вильгельма. Но Полани верил в ценность собственной теории даже после изучения им теории, предложенной Ленгмюром, которая показалось ведущим авторитетам более продвинутой. Однако он так и не смог убедить своих критиков аргументами в пользу своей концепции [13-15]. В то же время его теорию поддержали такие видные немецкие ученые как Г.М. Фрейндлих, Людвиг Берений (Ludwig Berényi) [25-27], Э. Берл (E. Berl) и В. Швебель (W. Schwebel) [28] и другие.

Немецкий физикохимик Г.М. Фрейндлих (1880-1941), на самом деле, был сторонником абсолютно всех основных теорий адсорбции начала 20 века - теории капиллярной конденсации Зигмонди, потенциальных теорий Эйкена и Полани, а также мономолекулярной теории Ленгмюра. Он их все проанализировал и описал в 
своей книге «Капиллярная химия» 1922 [24] и 1923 года издания (первое издание было выпущено в 1909 году).

В частности, в этой монографии Фрейндлих отмечал: «Аргументы сильно различаются в зависимости от того, адсорбционный слой состоит из нескольких слоев молекул или только одного. Я в настоящее время не способен однозначно выбрать между двумя теориями, их надо рассматривать более подробно. Поэтому, в качестве примера теории, постулирующей несколько слоев молекул, может быть принята теория Полани, а в качестве примера одного слоя - теория Ленгмюра» (Цит. по [24], стр. 122).

\section{Заключение}

Таким образом, в начале 20 века, после долгого периода первоначального накопления экспериментального материала по адсорбции в 19 веке, наступил этап бурного развития теоретических знаний в этой области. Началом послужили теория капиллярного гистерезиса (Р. Зигмонди, 1911), потенциальная теория адсорбции (А. Эйкен и М. Полани, 1914), а также монослойная (И. Ленгмюр, 1916-1918) и полимолекулярная (М. Полани, 1914) теории заполнения адсорбента адсорбатом. Их развитием стала полимолекулярная теория Брунауэра-Эммета-Теллера (1938). Появлению вышеуказанных теорий, несомненно, способствовали успехи в развитии химии и физики в начале 20 века, связанные с развитием химической термодинамики и теорий химической связи.

\section{Список литературы/References}

1. de Saussure T., Bibliothèque Britannique. 1812. Vol. 49, pp. 299-340.

2. de Saussure T., Annalen der Physik, 1814, Bd. 47, pp. 113-183.

3. Chappuis P., Annalen der Physik und Chemie, 1883, Bd. 19, pp. 21-38.

4. Chappuis P., Annalen der Physik und Chemie, 1879, Bd. 9, pp. 1-29.

5. Chappuis P., Annalen der Physik und Chemie. 1881. Bd. 12, pp. 161-181.

6. Magnus G. Poggendorf's Annalen, 1825, Bd. 79, pp. 81-88.

7. Magnus G., Annalen der Physik, 1853, Bd. 165, pp. 604-610.

8. Kayser H., Annalen der Physik, 1881, Bd. 12, pp. 526-588.

9. Kayser H., Annalen der Physik. 1881, Bd. 14, pp. 450-468.

10.Eucken A., Verhandlungen der Deutschen physikalischen Gesellschaftl 19141 Bd. 16, pp. 345-362.

11.Jo Nye. M. Michael Polanyi and his generation, Chicago, University of Chicago Press, 2011, 428 p.

12.Swan E, Urquhart A.R., Journal of Physical Chemistry, 1927, Vol. 31, pp. 251-276.
13.Pallo G. Ambix, 2010, Vol. 57, pp. 216230.

14.Wigner E.P., Hodgkin R.A., Biographical Memoirs of Fellows of the Royal Society, 1977, Vol. 23, pp. 413-448.

15.Polanyi M., Scienc, 1963, Vol. 141, pp. 1010-1013.

16.Polanyi M. Adsorption of gases by a solid non-volatile adsorbent. Ph.D. dissertation. Budapest. 1917.

17.Polanyi M., Verhandlungen der Deutschen physikalischen Gesellschaft, 1914, Bd. 16, pp. 1012-1016.

18.Polanyi M. Erwiderung an Herrn A., Zeitschrift für Elektrochemie und angewandte physikalische Chemie, 1922, Bd. 28, pp. 110-111.

19.London F., Polanyi M., Naturwissenschaften, 1930, Bd. 18, pp. 1099-1100.

20.Langmuir I., Journal of the American Chemical Society, 1916, Vol. 38, pp. 22212295.

21.Langmuir I., Journal of the American Chemical Society, 1917, Vol. 39, pp. 18481906.

22.Langmuir I., Journal of the American Chemical Society, 1918, Vol. 40, pp. 13611403. 
23.Palló G., Ambix, 2010, Vol. 57, pp. 216230.

24.Freundlich H. Capillarchemie. Leipzig: Akademische Verlagsgesellschaft, 1922, 1181 p.

25.Berényi L., Zeitschrift für Physikalische Chemie, 1920, Vol. 94, pp. 628-662.

Панарин Владислав Юрьевич - аспирант кафедры физической химии химического факультета Московского государственного университета им. М.В. Ломоносова, Москва

Баум Елена Анатольевна - к.Х.н., с.н.с. кафедры физической химии химического факультета Московского государственного университета им. М.В. Ломоносова, Москва

Ланин Сергей Николаевич - д.х.н., проф., заведующий лабораторией адсорбции и хроматографии кафедры физической химии химического факультета Московского государственного университета им. М.В. Ломоносова, Москва; тел.: (495) 939-19-26
26. Berényi L., Angewandte Chemie, 1922. Bd. 35 , pp. 237-238.

27.Berényi L., Zeitschrift für Physikalische Chemie, 1923, Vol. 105, pp. 55-72.

28. Berl E. Schwebel W., Angewandte Chemie, 1923, Bd. 36, pp. 541-545.

Panarin Vladislav Yu. - post-graduate student of department of physical chemistry of chemical faculty of Lomonosov Moscow State University, Moscow, e-mail: panarinvladyslav@gmail.com

Baum Elena A. - candidate of chemical sciences, senior researcher of department of physical chemistry of chemical faculty of Lomonosov Moscow State University, Moscow, e-mail: baumzai@mail.ru

Lanin Sergey N. - doctor of chemistry, professor, head of the laboratory of adsorption and chromatography of physical chemistry of chemical faculty of Lomonosov Moscow State University, Moscow, e-mail: silica2012@gmail.com 